\title{
MIDOT: A novel probe for monitoring high-current flat transmission lines
}

K. Omar, B. M. Novac, N. Graneau, P. Senior, I. R. Smith, and M. Sinclair

Citation: Review of Scientific Instruments 87, 125004 (2016);

View online: https://doi.org/10.1063/1.4971246

View Table of Contents: http://aip.scitation.org/toc/rsi/87/12

Published by the American Institute of Physics

\section{Articles you may be interested in}

Design of an accurate wireless data logger for vibration analysis with Android interface

Review of Scientific Instruments 87, 125003 (2016); 10.1063/1.4971326

An ultra-low noise, high-voltage piezo-driver

Review of Scientific Instruments 87, 124702 (2016); 10.1063/1.4969059

Design and characterization of microstrip based E-field sensor for GSM and UMTS frequency bands

Review of Scientific Instruments 87, 124703 (2016); 10.1063/1.4971316

Characterization of electrical noise limits in ultra-stable laser systems

Review of Scientific Instruments 87, 123105 (2016); 10.1063/1.4971852

Fiber-based modulated optical reflectance configuration allowing for offset pump and probe beams Review of Scientific Instruments 87, 124902 (2016); 10.1063/1.4967469

Sequential time interleaved random equivalent sampling for repetitive signal

Review of Scientific Instruments 87, 125106 (2016); 10.1063/1.4971877

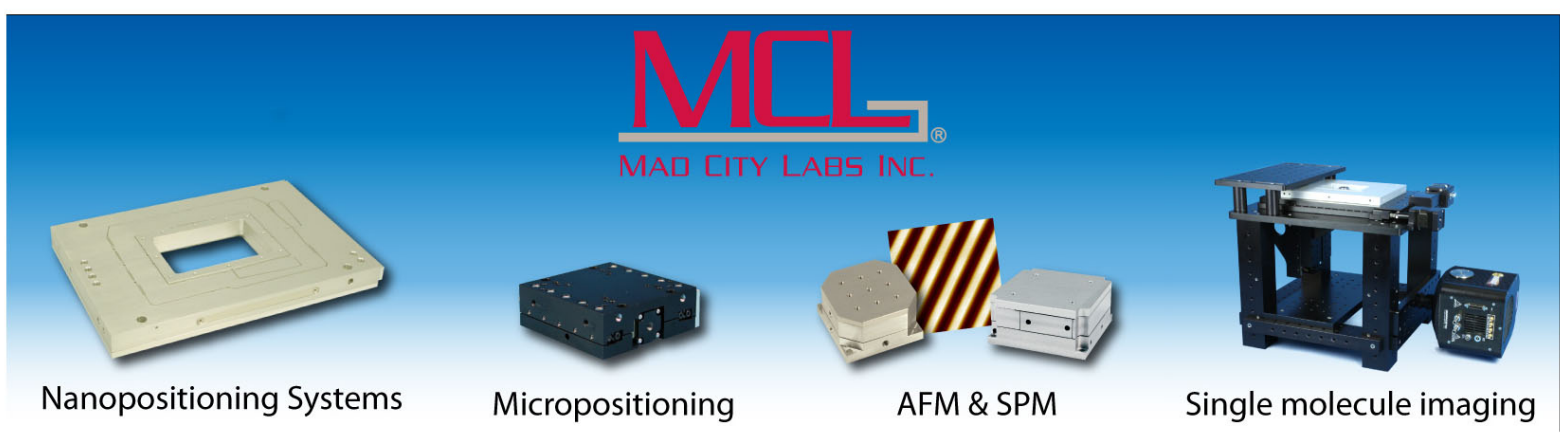




\title{
MIDOT: A novel probe for monitoring high-current flat transmission lines
}

\author{
K. Omar, ${ }^{1, a)}$ B. M. Novac, ${ }^{2, a), b)}$ N. Graneau, ${ }^{1}$ P. Senior, ${ }^{2}$ I. R. Smith, ${ }^{2}$ and M. Sinclair ${ }^{1}$ \\ ${ }^{1}$ Hydrodynamics Department, AWE, Aldermaston, Reading RG7 4PR, United Kingdom \\ ${ }^{2}$ Plasma and Pulsed Power Group, Loughborough University, Loughborough, \\ Leicestershire LE11 3TU, United Kingdom
}

(Received 1 September 2016; accepted 14 November 2016; published online 15 December 2016)

\begin{abstract}
A novel inductive probe, termed MIDOT, was developed for monitoring high-current flat transmission lines. While being inexpensive the probe does not require calibration, is resistant to both shock waves and temperature variations, and it is easy to manufacture and mount. It generates strong output signals that are relatively easy to interpret and has a detection region limited to a pre-defined part of the transmission line. The theoretical background related to the MIDOT probes, together with their practical implementation in both preliminary experimentation and high-current tests, is also presented in the paper. The novel probe can be used to benchmark existing 2D numerical codes used in calculating the current distribution inside the conductors of a transmission line but can also easily detect an early movement of a transmission line component. The probe can also find other applications, such as locating the position of a pulsed current flowing through a thin wire. [http://dx.doi.org/10.1063/1.4971246]
\end{abstract}

\section{INTRODUCTION}

High-energy high-current generators are extensively used in modern pulsed power research, with currents flowing through parallel-plate transmission lines having the familiar so-called "strip-line" conductor geometry of Figure 1. Representative examples include:

(i) high-velocity electromagnetic launchers such as the railgun, ${ }^{1}$

(ii) the "slapper" flyer plate impact shock-wave systems ${ }^{2}$ such as the recently developed AMPERE foil-flyer accelerator, $^{3}$

(iii) isentropic compression experimentation. ${ }^{4}$

Many other examples can be found in a number of worldwide facilities that employ large high-current capacitor banks coupled to a load via strip-lines, with an extreme example being SHIVA STAR. ${ }^{5}$ In many of these applications, an important issue is benchmarking the codes used in the complex numerical modelling of the system, which can predict the current distribution inside the transmission line conductors. However, it may also be necessary to detect the potential displacement of the strip-line conductors under heavy magnetic pressure loading. This may be related either to the application itself, as for any slapper-type of accelerator or as a (routine) safety procedure for the early detection of a possible failure of a high-current transmission line.

The direct measurement of the current distribution in strip-lines appears virtually impossible in practice. The most convenient but indirect procedure to obtain the current distribution is to measure the magnetic field distribution outside the line generated by the current. It is very important, however, to note that unfortunately the magnetic field distribution cannot

\footnotetext{
a)K. Omar and B. M. Novac contributed equally to this work.

b) Author to whom correspondence should be addressed. Electronic mail: b.m.novac@lboro.ac.uk
}

straightforwardly provide the current distribution unless a model is assumed and calculations are performed. Another issue is that the magnetic field distribution near the edge of a strip-line has a large gradient, requiring a very detailed spatial resolution that is difficult to obtain in practice with any existing magnetic field sensor. To illustrate this issue, a practical example is discussed below in detail.

The most convenient well-known technique for measuring magnetic fields uses magnetic pick-up (inductive) probes (fully described in books ${ }^{6}$ ). A simple numerical application detailed below shows, however, that this solution is impractical for strip-lines. The magnetic probe of Figure 1 is positioned in such a way that it is only influenced by the $\mathrm{Bx}$ component of the magnetic flux density. At any point $\mathrm{P}\left(\mathrm{x}_{\mathrm{p}}, \mathrm{y}_{\mathrm{p}}\right)$, the time rate-of change of $\mathrm{Bx}$ is ${ }^{3}$

$$
\begin{aligned}
\frac{d B_{x}}{d t}\left(x_{p}, y_{p}\right)= & \frac{\mu_{0}}{2 \pi \omega} \frac{d I}{d t}\left[\tan ^{-1}\left(\frac{x_{p}-w}{y_{p}}\right)-\tan ^{-1}\left(\frac{x_{p}}{y_{p}}\right)\right. \\
& \left.-\tan ^{-1}\left(\frac{x_{p}-w}{y_{p}-d}\right)+\tan ^{-1}\left(\frac{x_{p}}{y_{p}-d}\right)\right]
\end{aligned}
$$

where $w$ is the transmission line width, $d$ the spacing between the plates, and $d I / d t$ the time rate-of-change of the current $I$. During measurement, the magnetic probe generates a voltage $E M F_{m p}=-\sum_{k=1}^{N} V_{k}$, where $N$ is the number of its helical turns mounted on the cylindrical mandrel. The voltage induced in the $k$ th turn is calculated as $V_{k}=\int_{S_{k}} \frac{d B_{x}}{d t} d s$, with the integral performed over the corresponding active surface $S_{k}$. When a measurement is attempted near the strip line edge, the result is difficult to interpret because the $V_{k}$ voltages are unequal. Assume that for the strip line of Figure 1, $w=200 \mathrm{~mm}$ and $d=1 \mathrm{~mm}$ and the magnetic probe is wound on a mandrel of diameter $d_{m p}=1 \mathrm{~mm}$ and axial length $x_{m p}=1 \mathrm{~mm}$, positioned $y_{m p}=1 \mathrm{~mm}$ above the strip line edge. The number of turns is not important in this analysis. Simple calculations using Eq. (1) show that the ratio of the $d B_{x} / d t$ at the two 


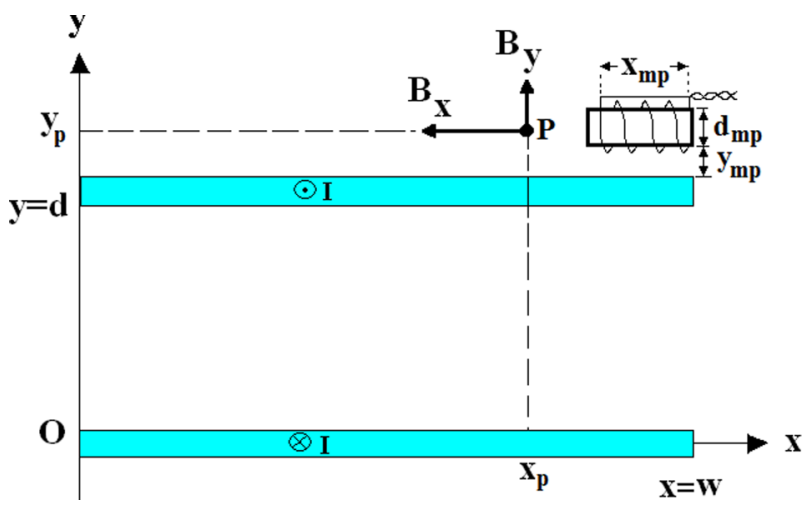

FIG. 1. Cross section geometry of a parallel-plate transmission line (schematic). A magnetic pick-up probe is located above the right hand edge of the line (not to scale).

extremities of the probe is extremely large (approximately $65)$ and the ratio $V_{1} / V_{N}$ between the voltages generated in the first and the last turns is correspondingly large. As a consequence, when the probe output is integrated it provides only the median value of the magnetic flux density, which is insufficiently precise for benchmarking. Clearly, only a probe with a micron-size axial length would be suitable for this task. To provide a usable voltage output would then require a relatively large number of turns, making the manufacture of such probes impractical. Furthermore, precise calibration is required and it is difficult to make the probe sensitive to only one component of the magnetic field.

More recently, a novel and remarkable microscopic size (active surface $400 \times 50 \mu \mathrm{m}$ ) scalar magnetic probe has been developed, based on the Colossal Magneto-Resistance (CMR) effect. ${ }^{7}$ An array of such probes was successfully used for studies of the current distribution inside the parallel transmission plates of a rail gun. ${ }^{7,8}$ However, there are a number of drawbacks that make its utilisation sometimes difficult since the probes can only accurately measure magnetic flux densities above $1.5 \mathrm{~T}$, are sensitive to strong mechanical shocks and temperature variation, and lastly, but not least importantly, they are expensive to replace in unavoidable destructive experiments such as those related to slapper accelerators.

From the above presentation, it is obvious that obtaining the current distribution from magnetic field distribution measurements is a very complicated and difficult task. An alternative and quite different technique was sought and this paper aims to present the most important aspects of this activity. The resulting novel probe developed is of micron size and simple to manufacture at very low cost. It is easy to install and provides results that can conveniently be used to benchmark numerical models which calculate the current distribution in parallel-plate transmission lines. The paper describes the characteristics of the probe, together with preliminary experimental results that highlight its main advantages. Results obtained in high-current strip-line tests that allow benchmarking of a 2D filamentary code are also presented.

\section{FILAMENTARY MODELLING AND THE NOVEL MIDOT PROBE}

The highly accurate 2D filamentary model of a stripline detailed previously ${ }^{3}$ is based on a numerical technique in which the strip-line conductors are divided into parallel, isolated filaments along the $O z$ axis, such that the filamentary currents flow uniquely in this direction (see Figure 2).

These currents form an electric circuit network for which Kirchhoff-type equations can be written, with the resulting system of first-order differential equations being numerically solved. By inspection, symmetry allows both conductors forming the transmission line (the upper termed $\mathrm{U}$ and the lower L, as in Figure 2) to be represented by two segments (left side termed L and right side R, as in Figure 2), through which the same current distribution flows as shown in Figure 2.

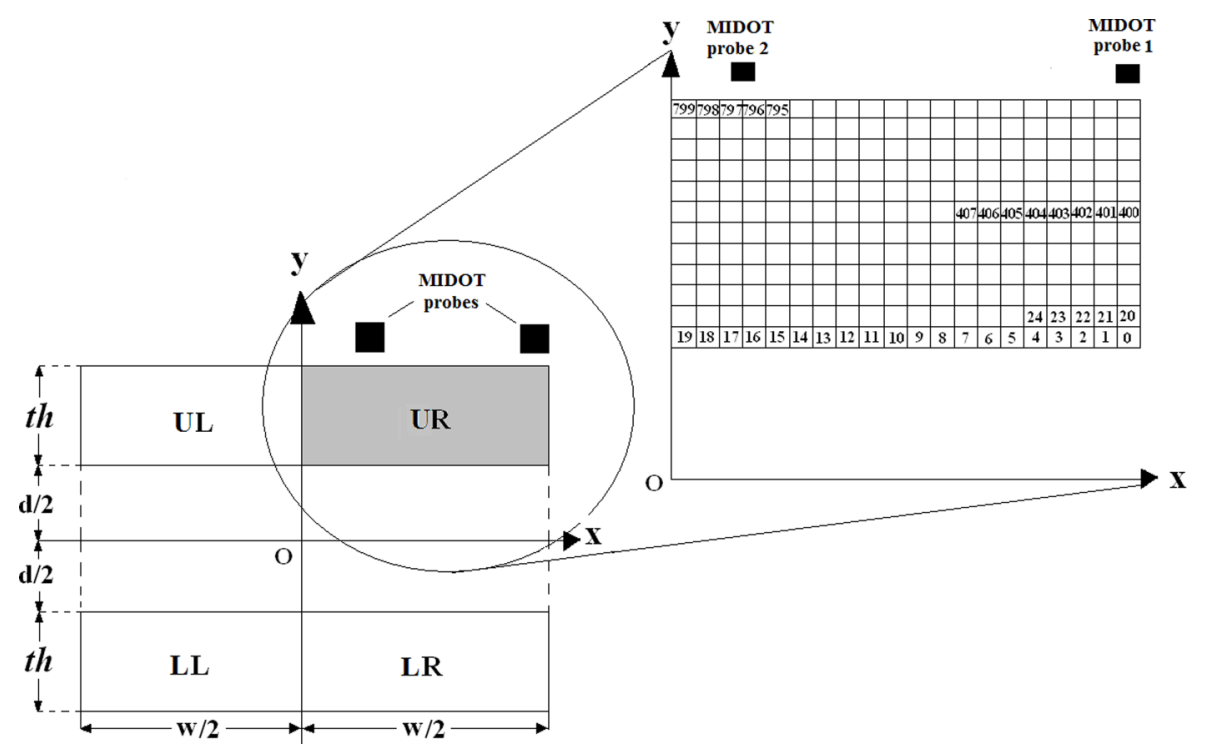

FIG. 2. Filamentary representation of a parallel-plate transmission line. For simplicity, the two conductors of the strip line, termed Upper and Lower, are shown as identical; by symmetry, only currents in one half (Right or Left) need to be calculated. Two external filaments, part of two MIDOT probes, are also shown as black squares. 
The technique therefore only requires calculation of the filamentary currents flowing through one segment of each conductor, which enables the total number of filamentary currents flowing to effectively be doubled.

The development of the novel technique began from an awareness that the mutual inductance between any two parallel filaments can be calculated extremely accurately by a simple and straightforward mathematical approach. ${ }^{3}$ Because the strip-line is represented by a collection of parallel imaginary filaments the idea is, as shown in Figure 2, to install similar but real filaments parallel to but outside the line, with the same type of procedure used to calculate the mutual inductances between those filaments and the transmission line. Each external filament effectively is an open circuit and, during a test, the time variation of the strip-line current induces an emf that can be expressed as follows:

$$
\begin{aligned}
E M F_{\text {filament }}= & \sum_{k=1}^{n}\left(M w_{k, f \text { flament }}^{U R}+M w_{k, \text { fliament }}^{U L}\right. \\
& \left.-M w_{k, \text { fliament }}^{L R}-M w_{k, \text { filament }}^{L L}\right) \frac{d I_{k}}{d t},
\end{aligned}
$$

where $n$ is the number of filaments into which each half of the transmission line conductors is divided and $M w_{k, \text { filament }}$ is the mutual inductance between the $k$ th filament of the line and the external filament (see Appendix). The different signs used with the various mutual inductances arise since the filamentary currents flow in opposite directions inside the two conductors of the transmission line. Finally $d I_{k} / d t$ is the time rate-ofchange of the $k$ th filamentary current. It is obvious from Eq. (2) that the integrated emf signal from the real filament is directly proportional to the virtual filamentary currents and therefore contains information about their spatial distribution since all the mutual inductances, due to their well-known position, are accurately known.

The external filament is part of the novel probe termed MIDOT, as its output depends only on the product of mutual inductances $(M)$ with their corresponding time rate-of-change of currents $(I-d o t)$. As will be presented later, once the emf impulses generated by a number of MIDOT probes are recorded, it allows benchmarking of the 2D filamentary model which can then provide the current distribution, as only one particular current distribution could generate the particular set of emfs.

\section{PRACTICAL IMPLEMENTATION OF THE NOVEL MIDOT PROBE}

In practice, the emf generated in the filament mounted above the transmission line is measured using a voltage probe with a sufficiently large input impedance (i.e., hundreds of $\mathrm{M} \Omega \mathrm{s}$ ), to allow the current flowing through its circuit to be neglected. In this condition no measurable influence is produced on the intense current flowing through the transmission line and, even more importantly, any number of such filaments can be mounted very close to one another without any measurable cross-talk. Preliminary tests (presented later) performed with an array of such filaments confirmed that this is the case.

A MIDOT probe is a two component assembly: one being a thin wire circuit in the form of a rectangle and the second a voltage sensor included in its circuit, as shown in Figure 3. Use of Cartesian coordinates as in Figures 1 and 2 shows that a MIDOT probe planar circuit includes two active filaments, each of length $z_{M}$. These need to be installed parallel to the direction of current flow through the transmission line (i.e., along the $\mathrm{Oz}$ axis), one directly above the region of interest and one at a distance from the strip-line (see Figure 3). To complete the circuit, these filaments are connected by two perpendicular parallel wires mounted parallel to the Ox axis, each of length $l_{\mathrm{M}}$. It can be demonstrated (see Appendix) that there is no emf generated in the connecting wires, hence the term "active filaments." In

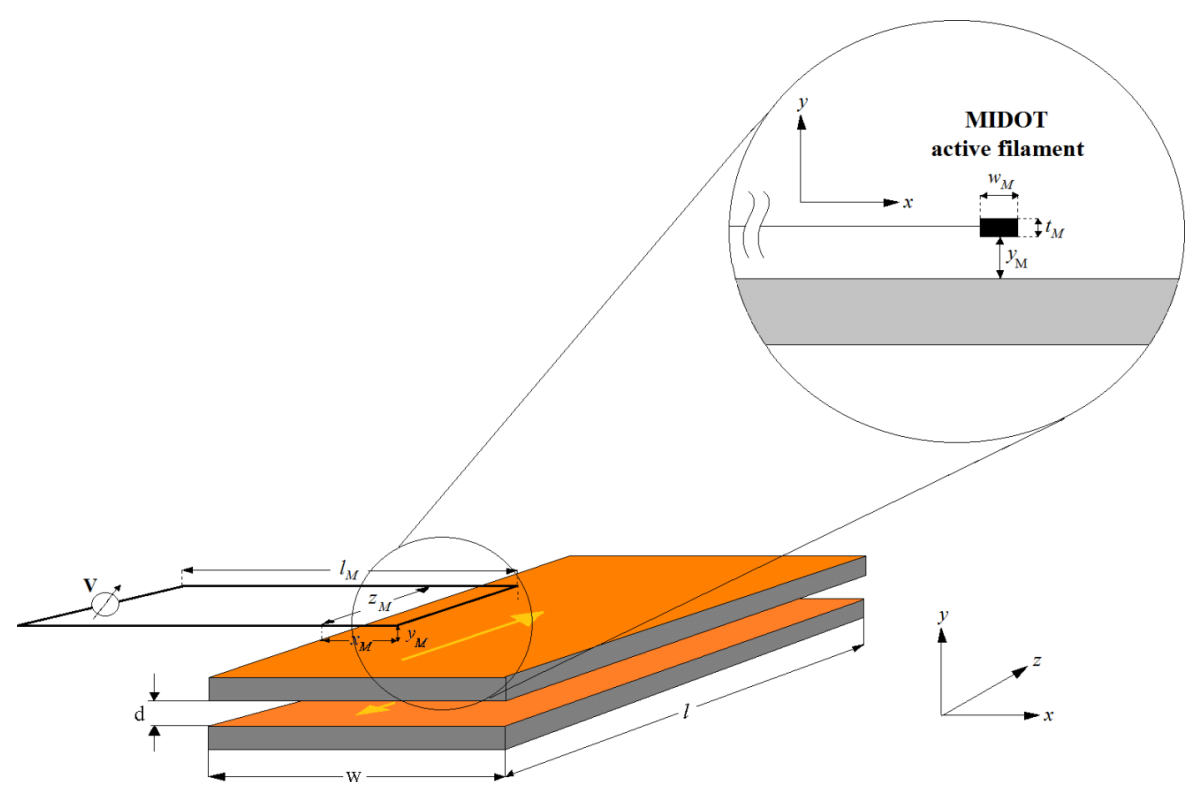

FIG. 3. Parallel-plate transmission line with a MIDOT probe mounted above (schematic). Inset shows details of the MIDOT active filament mounted above the line. 
theory, as the length $l_{\mathrm{M}}$ tends to infinity, only the filament positioned above the strip-line remains active. In reality, however, for practical reasons, $l_{\mathrm{M}}$ cannot be made too long and therefore the two active filaments experience opposing induced emfs.

To understand the functioning of the MIDOT probe and what its design requirements are, it is necessary to calculate the mutual inductances between the active filaments of the probe and the strip line filaments (see Eq. (2)). This task is detailed in the Appendix.

As shown in Figure 3, the MIDOT active filament positioned above the transmission line (with details in the inset) has width $w_{M}$, thickness $t_{M}$, and an active length $z_{M}$, which depend on the length of the region along the transmission line that is under investigation. Somewhat unexpectedly, calculation of the mutual inductance between the active MIDOT filament and the transmission line (detailed in the Appendix) revealed that only the part of the transmission line corresponding to the length $z_{M}$, and facing the MIDOT probe, plays a major role in the measurement. A MIDOT probe therefore provides very localized results, which could be important in some applications, allowing the experimental study of a limited part of a long transmission line. As the length under study is usually of the order of a few hundreds of $\mathrm{mm}$ or longer, an active filament length can be easily measured with a precision better than $1 \%$.

The conductor used in the construction of a MIDOT active filament can be round, with a diameter of only a few tens of $\mu \mathrm{m}$, but this is not necessarily the best solution. As described in our previous work, ${ }^{3}$ when filamentary modelling of a stripline is undertaken, the available computer memory limits the number of filaments to a rather low figure. Because of the thin and wide geometry of the flyer plate, the filaments have a rectangular cross section with one side many tens of times longer than the other, very much like a very thin strip.

It is sometimes convenient to choose the (real) MIDOT active filament cross section geometry $\left(w_{M}\right.$ and $\left.t_{M}\right)$ to be precisely the same as that of the (virtual) strip-line filaments used in the modelling. In this way the details and precision of the numerical calculations can match those of the measurement. In most numerical cases studied, this meant using filaments with widths between $2 \mathrm{~mm}$ and $3 \mathrm{~mm}$ and thicknesses between $30 \mu \mathrm{m}$ and $100 \mu \mathrm{m}$. Fortunately thin copper strips with precisely such dimensions are commercially available (for example, $w_{M}=3.5 \mathrm{~mm}$ and $t_{M}=60 \mu \mathrm{m}$ ) having as a bonus one side coated with an acrylic adhesive for easy mounting. The thickness of the MIDOT probe is therefore well-defined and does not need measurement.

The most challenging requirement is to position the MIDOT circuit in a plane parallel to the strip-line and at a height known with a relatively high precision. Calculations presented in the Appendix demonstrate that in most practical cases the error in positioning the active filaments above the strip-line should be less than $300 \mu \mathrm{m}$. This will ensure an error between the calculated mutual inductances and their real value of much less than 5\%, which is considered to be acceptable. In practice, the required precision was easily achieved by constructing the MIDOT circuit using self-adhesive copper strips mounted on a flat plastic plate of thickness $y_{M}$.

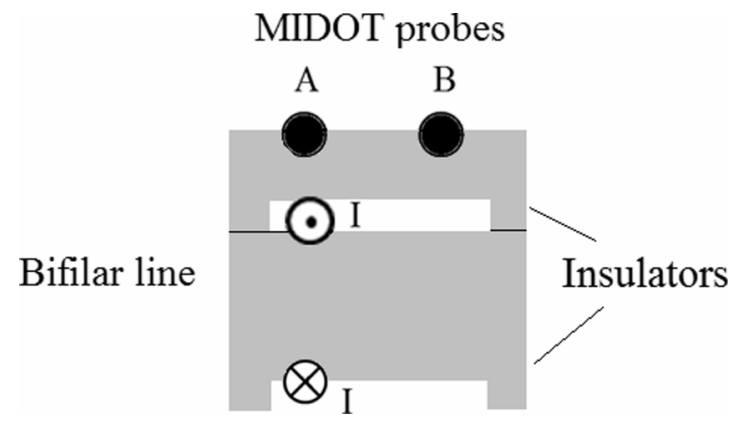

FIG. 4. Cross section of parallel-wire (bifilar) transmission line, through which a current I flows (schematic). The active filaments of two MIDOT probes, A and B, are shown mounted above the line at known heights set by the thickness of insulators.

As a conclusion to this paragraph, the most important characteristics of the novel MIDOT probe are summarized as follows:

(i) It does not require calibration.

(ii) It is resistant to shock waves and temperature variations.

(iii) It is very low in cost.

(iv) It is easy to manufacture.

(v) It is easy to mount and use.

(vi) It has a strong output signal that is relatively easy to interpret.

(vii) Its detection region is limited to a pre-defined part of the strip-line.

\section{EXPERIMENTAL RESULTS AND THEIR ANALYSIS}

\section{A. Preliminary studies}

A number of preliminary studies were undertaken to both confirm the important characteristics and to investigate the

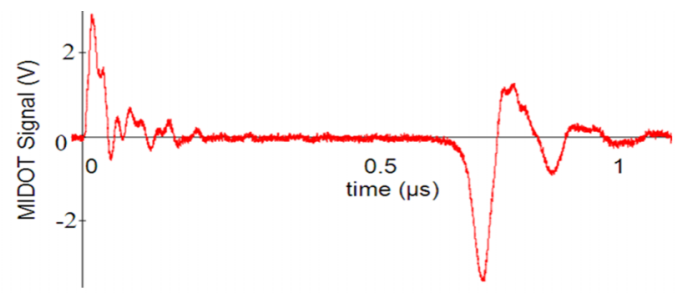

(a)

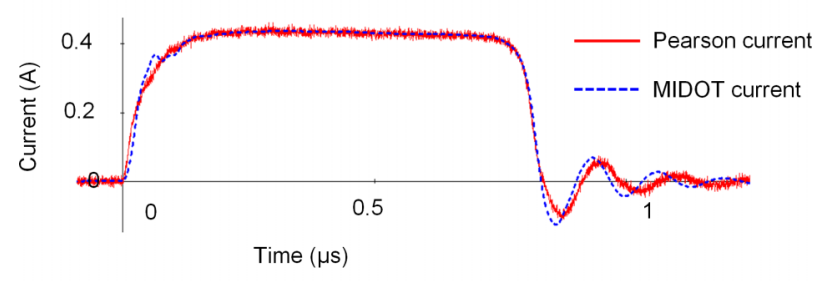

(b)

FIG. 5. (a) Oscilloscope signal generated by a MIDOT probe during preliminary testing and (b) comparison between the current flowing through the transmission line measured by a current transformer and the integrated current calculated from (a) using the MIDOT-transmission line equivalent mutual inductance. Small differences between the two signals are a consequence of differences in the voltage probe and current transformer bandwidths (see text). 


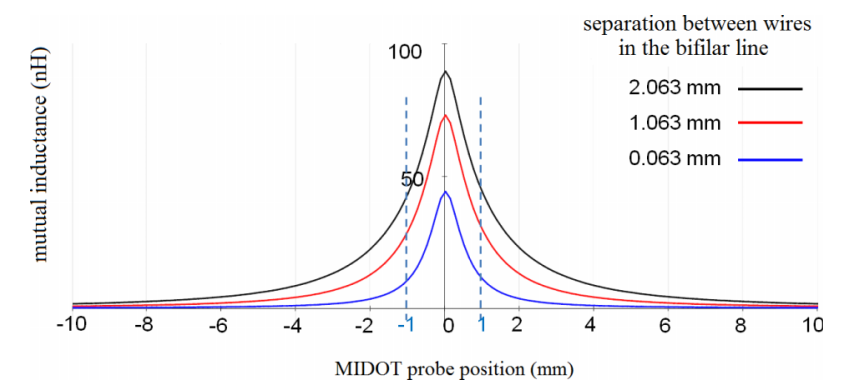

FIG. 6. Variation of the MIDOT-bifilar line mutual inductance with the horizontal and vertical separation between the probe and the bifilar line. The origin corresponds to the position of the bifilar line.

precision of measurement that can be obtained in practice with MIDOT probes. To simplify the interpretation of the results, the basic arrangement for these experiments, schematically shown in Figure 4, uses a thin wire parallel transmission line (a bifilar line) instead of the parallel plate transmission line. This arrangement is equivalent to a pair of virtual filaments used in the numerical modelling of a strip-line. The current flowing through the bifilar lines was generated using a commercial pulse generator ${ }^{9}$ and measured using a $200 \mathrm{MHz}$ bandwidth current transformer. ${ }^{10}$ Figure 5(a) shows a typical oscilloscope recording of the voltage $V(t)=M_{\text {MIDOT }} \frac{d I}{d t}$, generated by a MIDOT probe circuit and measured using its dedicated $400 \mathrm{MHz}$ bandwidth voltage probe, ${ }^{11}$ where the MIDOTtransmission line equivalent mutual inductance is calculated from $M_{M I D O T}=\left(M_{u p, 1}-M_{u p, 2}\right)-\left(M_{l o w, 1}-M_{l o w, 2}\right)$ (see the Appendix), with "up" and "low" representing the two wires of the transmission line and " 1 " and " 2 " the two active filaments of the MIDOT probe. Figure 5(b) presents a comparison

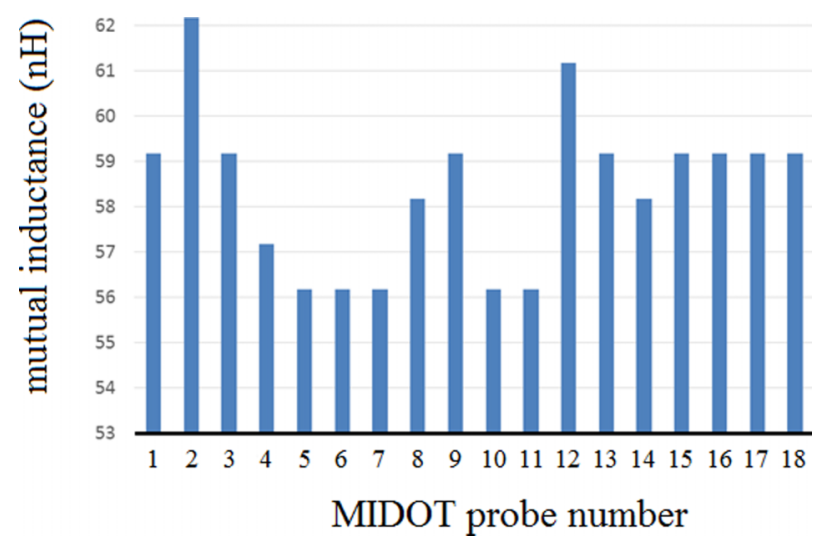

FIG. 8. Typical experimentally determined mutual inductance between probes in a MIDOT array and a strip-line. The mutual inductances are inside the domain $59 \mathrm{nH} \pm 5 \%$.

between the current $I(t)$ measured by the current transformer and the current obtained from integrating the MIDOT voltage. It is common practice with any type of inductive probe, such as a Rogowski coil or a magnetic pick-up, to use them in pairs, with the two probes mounted such that their outputs provide opposite polarity signals. This technique provides cancellation of any possible perturbing capacitive pickup effects with the signals generated by a MIDOT probe alternately measured with its voltage probe coupled in two ways, such that the output polarity is either positive or negative and $V_{M I D O T}^{ \pm}$ $= \pm M_{\text {MIDOT }} \frac{d I}{d t}+V_{p}$. In the two cases, the perturbing voltages $\mathrm{Vp}$ are co-phasal, while the magnetically induced signals are anti-phasal. The influence of any capacitive perturbation is cancelled, since $V=\frac{V_{M I D O T}^{+}-V_{M I D O T}^{-}}{2}$. In practice, the technique

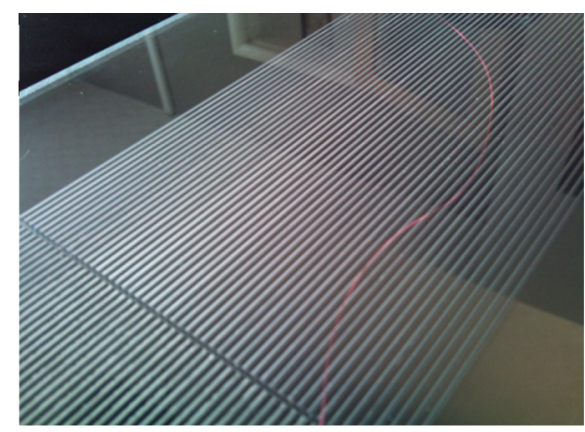

(a)

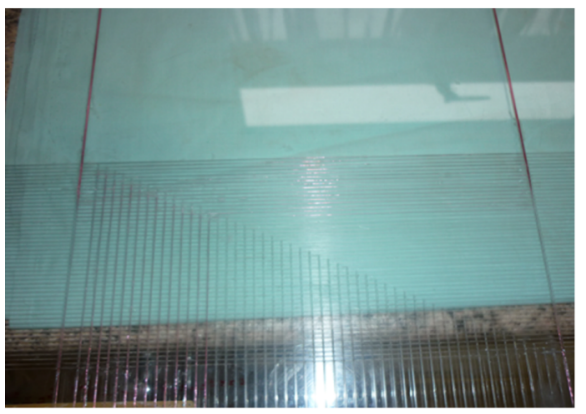

(c)

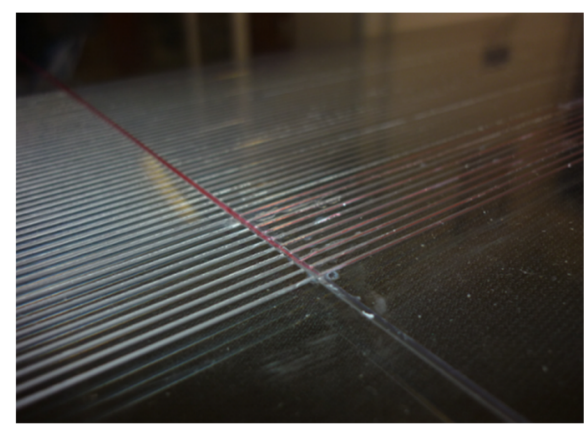

(b)

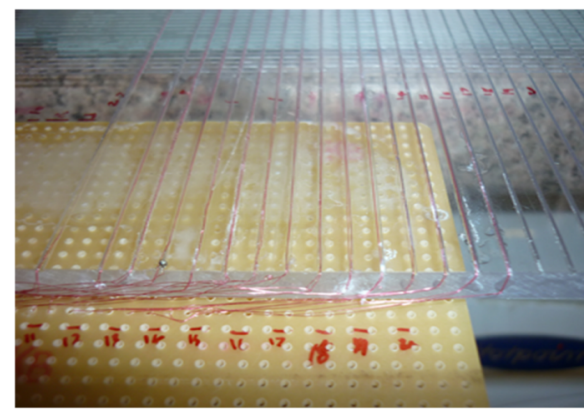

(d)

FIG. 7. Manufacture of a MIDOT array. (a) Machined acrylic template. (b) First few filaments positioned and fixed into place. (c) Completed MIDOT circuits. (d) Circuit ends connected to a PCB board to ensure robust electrical and mechanical connections. 


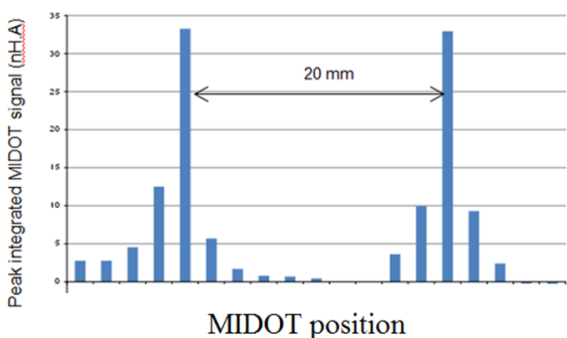

MIDOT position

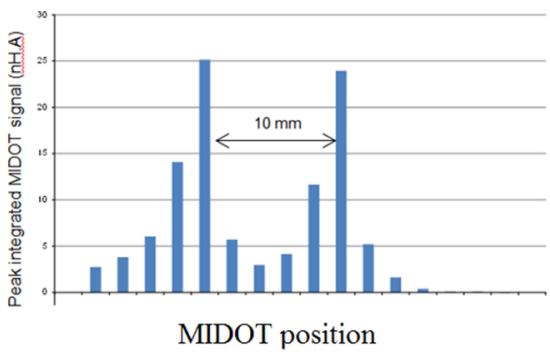

a)
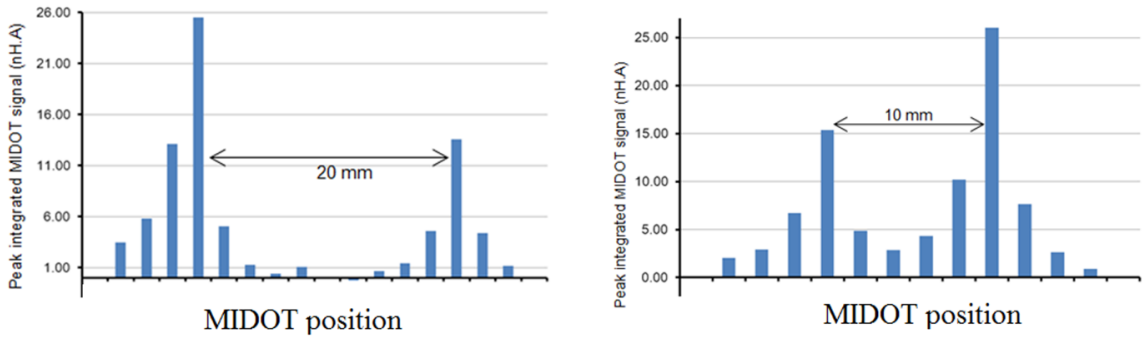

b)

FIG. 9. Signal intensities from the various probes of a MIDOT array, positioned above two parallel bifilar lines separated by $20 \mathrm{~mm}$ and $10 \mathrm{~mm}$, clearly showing: (a) the position of two parallel bifilar lines through which the same pulsed current flows; (b) same as (a) but with the lines pulsed with currents of different intensities.

also substantially removes electromagnetic noise, leading to the clean signal shown in Figure 5. Space does not permit a detailed presentation of all types of preliminary investigations and in what follows only the most important results are mentioned.

\section{Investigating the MIDOT probe spatial resolution}

The experimental performance of the MIDOT arrangement was assessed by changing the MIDOT position with respect to a bifilar line position. As evident from Figure 6,

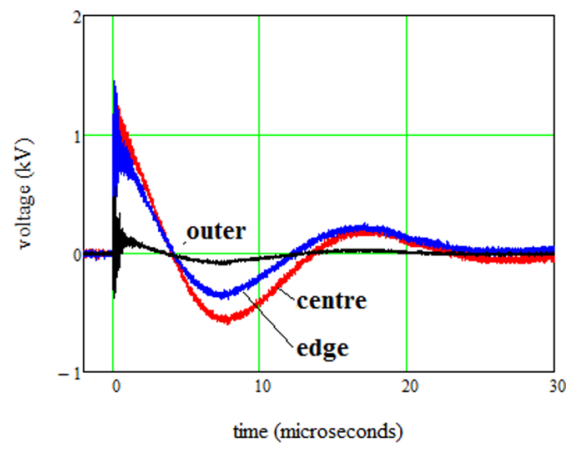

b)

a)

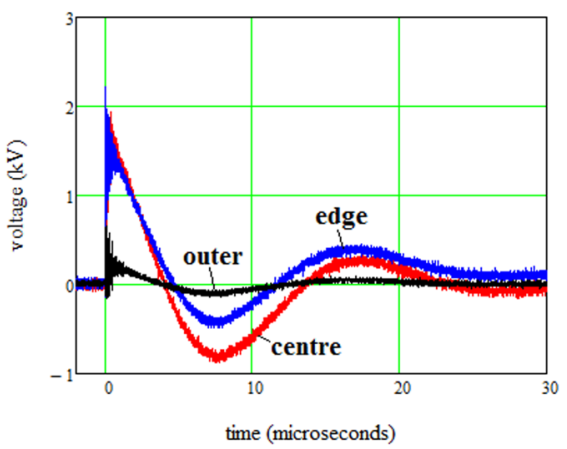

c)

FIG. 10. Signals recorded from three MIDOT probes for the bank charged to: (a) $6 \mathrm{kV}$, (b) $12 \mathrm{kV}$, and (c) $18 \mathrm{kV}$. 
the MIDOT probe is sensitive only to the bifilar line currents flowing within a few $\mathrm{mm}$ of the line, i.e., pulsed currents flowing further away do not induce any appreciable voltage in the probe circuit. This means that, in the case of an array of parallel MIDOT probes, different pulsed currents flowing through different parallel bifilar lines produce only localised signals, allowing detection of their position. This idea was tested in practice with the results presented below.

\section{Tests with MIDOT array}

A MIDOT array was manufactured during the developmental phase from $60 \mu \mathrm{m}$ diameter enameled wires mounted $2 \mathrm{~mm}$ apart inside parallel grooves machined into a plastic plate (Figure 7). After the wires were glued in position, the high-precision MIDOT array assembly was placed above the bifilar line such that the distance between the bifilar line and each of the MIDOT filaments was known with sufficient accuracy. Such an array was later used for detecting the current dynamics of an accelerated flyer. ${ }^{3}$ The array could withstand the impact of the flyer, without the risk of a galvanic connection between the flyer high-voltage circuit and the
MIDOT circuits attached to sensitive oscilloscope outputs. Flyer related results will be presented later. Preliminary tests, using the MIDOT array probing either a strip line or various pairs of bifilar line arrangements connected to the same voltage pulse generator, proved that:

(i) for adjacent MIDOT probes positioned $10 \mathrm{~mm}$ apart, no cross-talking effects could be detected,

(ii) the experimentally obtained MIDOT-strip line mutual inductances are close to the theoretically predicted figures, with errors up to $5 \%$ (Figure 8). This confirms that, with carefully positioned MIDOT probes, no calibration is required (see the Appendix),

(iii) a MIDOT array can detect both the relative position and intensity of two (or multiple) pulsed currents flowing through independent parallel bifilar lines (Figure 9).

\section{B. High-current tests}

\section{Detection of the early movement of strip-line components}

The strip line used in these tests has $l=0.5 \mathrm{~m}$, w $=150 \mathrm{~mm}$, and $\mathrm{d}=2 \mathrm{~mm}$ and is part of the Quattro bank

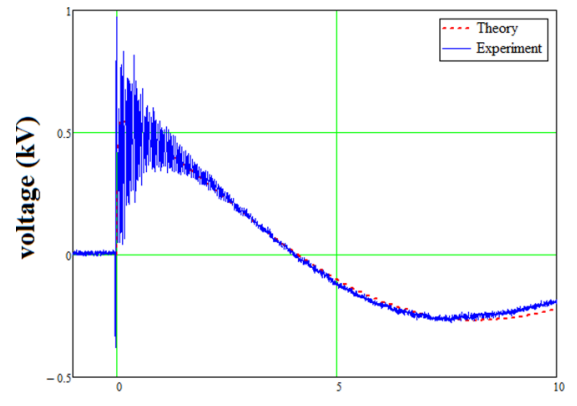

time (microseconds)

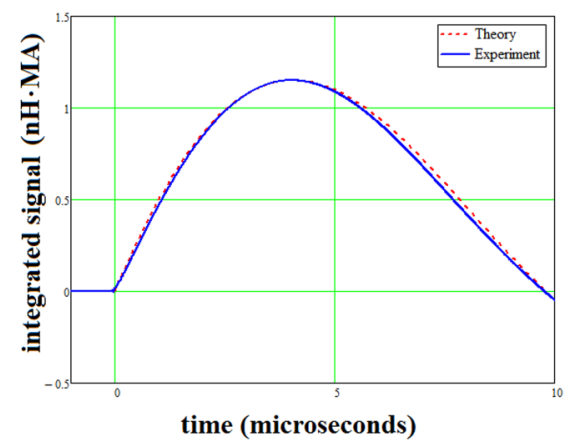

a)

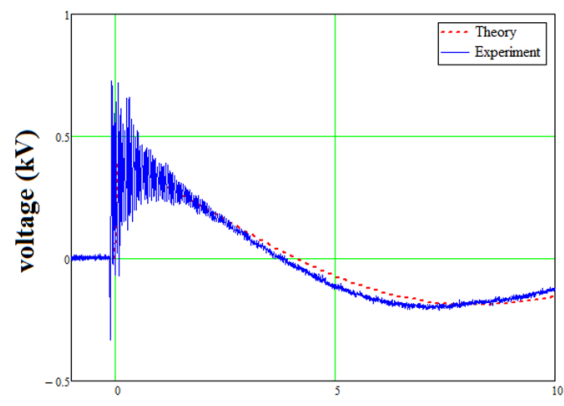

time (microseconds)

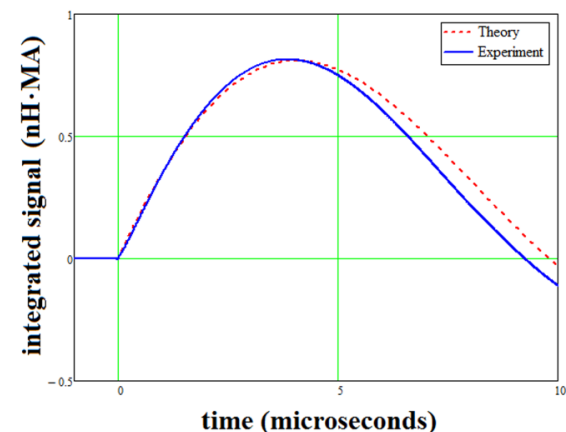

b)

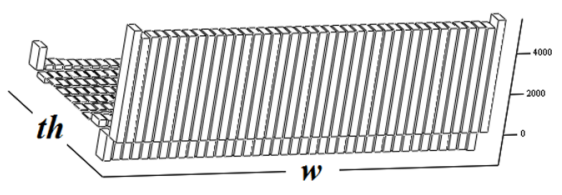

c)

FIG. 11. Results from (a) edge and (b) centre MIDOT probes compared with theoretical predictions. In both (a) and (b) the upper trace shows the emf generated by each probe, whilst the lower presents the corresponding integrated signal; (c) modelled current distribution inside the strip-line $3 \mu$ s from the beginning of the discharge (drawing not to scale, $w$ and $t h$ as in Fig. 2). 

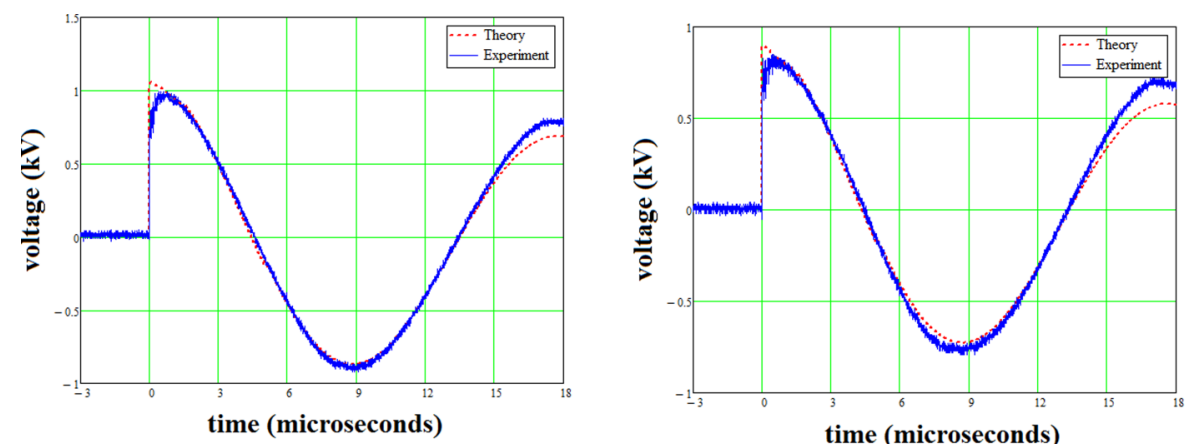

time (microseconds)

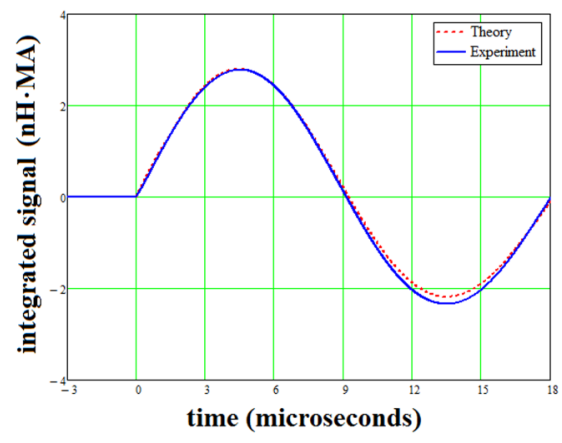

a)

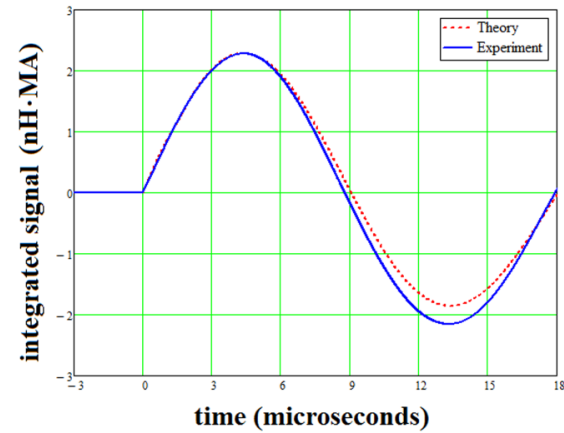

b)

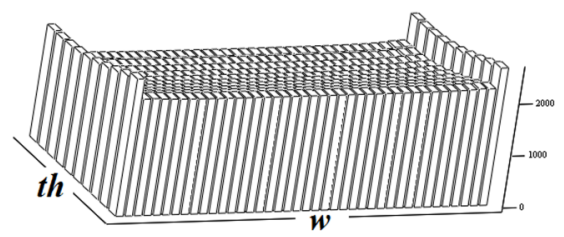

c)

FIG. 12. Results from (a) edge and (b) centre MIDOT probes compared with theoretical predictions. In both (a) and (b) the upper trace shows the emf generated by each probe, whilst the lower presents the corresponding integrated signal; (c) modelled current distribution inside the strip-line $7 \mu$ s from the beginning of the discharge (drawing not to scale, $w$ and $t h$ as in Fig. 2).

described in a previous work. ${ }^{12}$ The MIDOT probes were made from copper strips, having $w_{M}=3.5 \mathrm{~mm}, t_{M}=60 \mu \mathrm{m}$, $z_{M}=290 \mathrm{~mm}$, and $l_{M}=100 \mathrm{~mm}$, and attached to a $2 \mathrm{~mm}$ thick acrylic plate in contact with the upper conductor of the strip-line (i.e., $y_{M}=2 \mathrm{~mm}$ ). In some experiments, two or even three MIDOT probes were used, having the characteristics: centre probe $x_{M}=75 \mathrm{~mm}$, edge probe $x_{M}=0 \mathrm{~mm}$, and outer probe $x_{M}=-37.5 \mathrm{~mm}$.

A $5 \mathrm{~mm}$ layer of rubber is installed above the strip-line and compressed by a heavy inertial plate. The role of this layer is to attenuate as much as possible any upper plate movement, not allowing the accelerated conductor to be violently reflected backwards and thus preventing any possible damage to the insulating Mylar sheets positioned between the two copper conductors forming the strip-line, a technique described in an unpublished report. ${ }^{13}$ For the section of the line where MIDOT probes were installed, the rubber and the inertial mass were placed above the acrylic plate containing the probe circuits. The aim of these tests was to investigate if the MIDOT probes can detect even very limited upper plate movement.

Three tests were performed with the bank successively charged to $6 \mathrm{kV}, 12 \mathrm{kV}$, and finally $18 \mathrm{kV}$, progressively increasing the peak current from less than 0.3 MA to 0.8 MA and thereby the force acting on the strip line. The results, presented in Figure 10, show the effects of the conductor dynamics: the signals from the three MIDOT probes have the same first and second zero-crossing times for the lowest charging voltage (Figure 10(a)), when the forces acting on the strip-line are relatively small, but slightly different zero-crossing times for $12 \mathrm{kV}$ (Figure 10(b)) and finally different zero-crossing times at the highest charging voltage (Figure 10(c)). This is a clear indication of the changes in the values of the mutual inductance between the three probes and the strip-line due to the upper plate dynamics under heavy loading. It is thus clear that MIDOT probes can successfully be used to detect any early movement of a transmission line component.

\section{Benchmarking the 2D filamentary model}

Figures 11 and 12 present experimental results obtained in two different transmission line experiments, each using two MIDOT probes, and compare these with corresponding predictions from $2 \mathrm{D}$ filamentary modelling. The input data for the two tests are given below. 
Data for Figure 11: Capacitor bank: $\mathrm{C}=210 \mu \mathrm{F}$ charged to $6.4 \mathrm{kV}$ and generating a peak current of $0.3 \mathrm{MA}$. The bank circuit has a self-inductance of $39 \mathrm{nH}$ and a resistance of $1 \mathrm{~m} \Omega$ and includes a supplementary damping resistor made from thin stainless steel sheet with a room temperature resistance of $7.2 \mathrm{~m} \Omega$. Transmission line made from copper with dimensions: $w=150 \mathrm{~mm}$, th $=0.5 \mathrm{~mm}$, and $d=2 \mathrm{~mm}$. MIDOT probes: Centre and edge, as described in Sec. IV B 1 above.

Data for Figure 12: Capacitor bank: $\mathrm{C}=210 \mu \mathrm{F}$ charged to $13.3 \mathrm{kV}$ and generating a peak current of $0.6 \mathrm{MA}$. The bank circuit has a self-inductance of $33 \mathrm{nH}$ and an initial resistance of $1 \mathrm{~m} \Omega$, and includes a supplementary damping resistor made from thin stainless steel sheet with a room temperature resistance of $7.2 \mathrm{~m} \Omega$. Transmission line of aluminum with dimensions: $w=152 \mathrm{~mm}, t h=0.3 \mathrm{~mm}$, and $d=2.15 \mathrm{~mm}$. The MIDOT probes have $w_{M}=5 \mathrm{~mm}, t_{M}=60 \mu \mathrm{m}, y_{M}=2 \mathrm{~mm}$, and $z_{M}=290 \mathrm{~mm}$. The centre probe has $l_{M}=83.5 \mathrm{~mm}$ and $x_{M}=75 \mathrm{~mm}$ and the edge probe has $l_{M}=20 \mathrm{~mm}$ and $x_{M}$ $=0 \mathrm{~mm}$. In both Figures 11 and 12 the theoretical predictions are very close to the experimental results until the upper part of the transmission line starts to move, a phenomenon not considered in the calculations. In both figures, the predicted current distribution is also shown.

It is interesting to note that MIDOT probes survived and therefore could be used in many consecutive high-current tests.

\section{CONCLUSIONS}

A novel type of sensor termed MIDOT has been introduced and its characteristics highlighted, making it a valuable diagnostic tool for the high-current transmission lines used in many modern high-energy pulsed power generators. Without requiring calibration, the sensor can easily detect any movement of the transmission line elements and allow benchmarking of the codes used to calculate the current distribution in a strip-line. Other properties of the new probe, such as finding the position of a pulsed current flowing through a thin wire, can also find applications in other domains.

\section{ACKNOWLEDGMENTS}

(C) Crown copyright 2016. Reproduced with the permission of the Controller of Her Majesty's Stationery Office/Queen's Printer for Scotland and AWE.

\section{APPENDIX: MUTUAL INDUCTANCE BETWEEN A MIDOT PROBE AND A BIFILAR TRANSMISSION LINE}

A MIDOT probe can be used with either a strip line or a bifilar line. As the mutual inductance between the probe and a strip line can be calculated as a collection of mutual inductances with a large number of parallel bifilar lines (see the work on filamentary modelling ${ }^{3}$ ), in what follows only the technique used to calculate the mutual inductance between the MIDOT and a bifilar line is presented. The exact formula for the mutual inductance between a pair of very thin wires separated by distance $d$ and having length $l$ is ${ }^{14}$

$M w(l, d)=\frac{\mu_{0} l}{2 \pi}\left[\ln \left(\frac{l+\sqrt{l^{2}+d^{2}}}{d}\right)-\frac{\sqrt{l^{2}+d^{2}}}{l}+\frac{d}{l}\right]$.

When the wires are close, their cross section becomes important and $d$ must be replaced by the geometrical mean distance (GMD) between the two wires. ${ }^{3}$

In practice transmission lines are long with respect to the length of the MIDOT active filament (see text), with the probe being mounted parallel to anywhere along the line and placed in a different parallel plane, as shown in Figure 13. In such a condition, the mutual inductance between each of the two bifilar wires of length $l$ and the two MIDOT active filaments of length $z_{M}$ can be calculated, the passive elements (those which are perpendicular) can be ignored for reasons that can be found in the literature, ${ }^{14}$

$$
\begin{aligned}
& M_{u p, 1}=\frac{1}{2}\left[\begin{array}{l}
\left(M w\left(z_{M}+k, \sqrt{x_{M}^{2}+y_{M}^{2}}\right)+M w\left(l-k, \sqrt{x_{M}^{2}+y_{M}^{2}}\right)\right)- \\
\left(M w\left(k, \sqrt{x_{M}^{2}+y_{M}^{2}}\right)+M w\left(l-k-z_{M}, \sqrt{x_{M}^{2}+y_{M}^{2}}\right)\right)
\end{array}\right], \\
& M_{u p, 2}=\frac{1}{2}\left[\begin{array}{l}
\left(M w\left(z_{M}+k, \sqrt{\left(x_{M}+l_{M}\right)^{2}+y_{M}^{2}}\right)+M w\left(l-k, \sqrt{\left(x_{M}+l_{M}\right)^{2}+y_{M}^{2}}\right)\right)- \\
\left(M w\left(k, \sqrt{\left(x_{M}+l_{M}\right)^{2}+y_{M}^{2}}\right)+M w\left(l-k-z_{M}, \sqrt{\left(x_{M}+l_{M}\right)^{2}+y_{M}^{2}}\right)\right)
\end{array}\right], \\
& M_{l o w, 1}=\frac{1}{2}\left[\begin{array}{l}
\left(M w\left(z_{M}+k, \sqrt{x_{M}^{2}+\left(y_{M}+d\right)^{2}}\right)+M w\left(l-k, \sqrt{x_{M}^{2}+\left(y_{M}+d\right)^{2}}\right)\right)- \\
\left(M w\left(k, \sqrt{x_{M}^{2}+\left(y_{M}+d\right)^{2}}\right)+M w\left(l-k-z_{M}, \sqrt{x_{M}^{2}+\left(y_{M}+d\right)^{2}}\right)\right)
\end{array}\right], \\
& M_{l o w, 2}=\frac{1}{2}\left[\begin{array}{l}
\left(M w\left(z_{M}+k, \sqrt{\left(x_{M}+l_{M}\right)^{2}+\left(y_{M}+d\right)^{2}}\right)+M w\left(l-k, \sqrt{\left(x_{M}+l_{M}\right)^{2}+\left(y_{M}+d\right)^{2}}\right)\right)- \\
\left(M w\left(k, \sqrt{\left(x_{M}+l_{M}\right)^{2}+\left(y_{M}+d\right)^{2}}\right)+M w\left(l-k-z_{M}, \sqrt{\left(x_{M}+l_{M}\right)^{2}+\left(y_{M}+d\right)^{2}}\right)\right)
\end{array}\right] .
\end{aligned}
$$




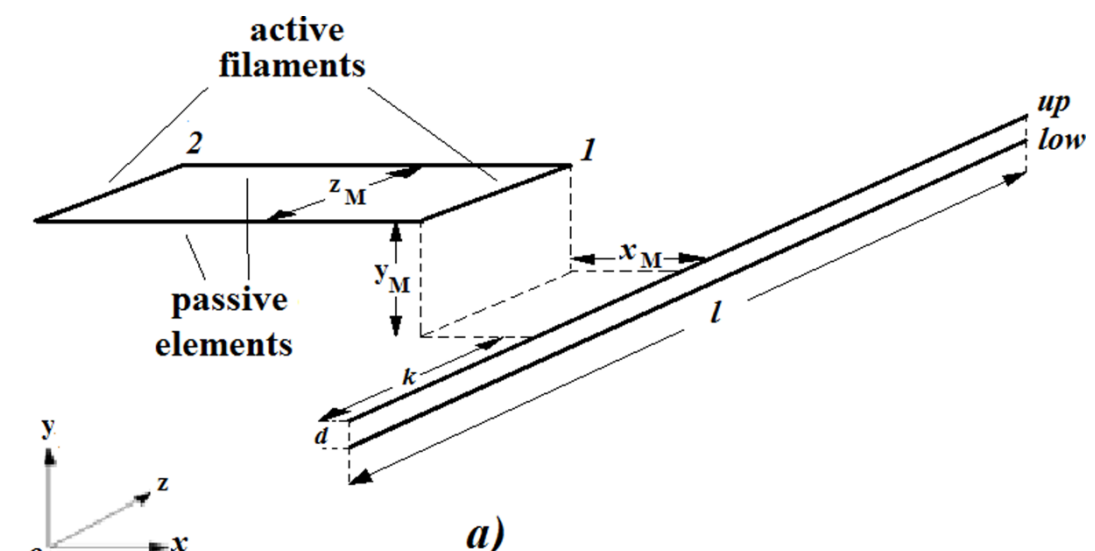

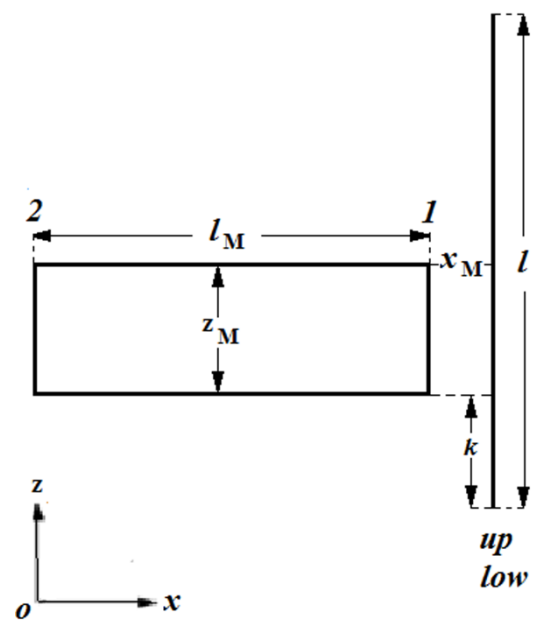

b)

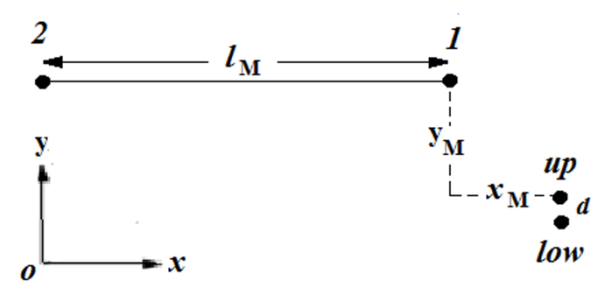

c)

FIG. 13. MIDOT probe with active filaments 1 and 2, positioned near a bifilar line made from parallel wires up and low (a) 3D view; for clarity (b) shows an $x O z$ view and (c) shows an $x O y$ view.

\section{Demonstration 1}

The mutual inductance between a MIDOT probe and a bifilar line can be calculated using only the interactions between each MIDOT active filament and each of the two wires of the bifilar line as follows:

$M_{\text {MIDOT }}=\left(M_{u p, 1}-M_{u p, 2}\right)-\left(M_{l o w, 1}-M_{l o w, 2}\right)$

where the - signs indicate that the direction of the current flow is reversed.

The demonstration is straightforward and is based on calculating $M_{M I D O T}$ from the magnetic flux $\Phi_{M I D O T}$ produced by the bifilar line current $I$ and captured by the MIDOT circuit: $M_{M I D O T}=\frac{\Phi_{M I D O T}}{I}$. Using cylindrical coordinates with the origin at one edge of a wire, the magnetic flux density generated at any point $(r, z)$ by a straight wire of length $l$ through which a current $I$ flows is given by ${ }^{15} B w(l, r, z)=\frac{\mu_{0} I}{2 \pi r}\left[\frac{l-z}{\sqrt{(l-z)^{2}+r^{2}}}+\frac{z}{\sqrt{z^{2}+r^{2}}}\right]$.

With this expression, the mutual inductance between the MIDOT circuit and the bifilar line can be easily calculated as

$$
\begin{aligned}
M_{\text {MIDOT }}= & \int_{x_{M}}^{x_{M}+l_{M}}\left[\int _ { k } ^ { k + z _ { M } } \left(\frac{B w\left(l, \sqrt{r^{2}+y_{M}^{2}}, z\right)}{\sqrt{r^{2}+y_{M}^{2}}}\right.\right. \\
& \left.\left.-\frac{B w\left(l, \sqrt{r^{2}+\left(y_{M}+d\right)^{2}}, z\right)}{\sqrt{r^{2}+\left(y_{M}+d\right)^{2}}}\right) d z\right] r d r,
\end{aligned}
$$

where for simplicity $I=1 \mathrm{~A}$. It is easy to demonstrate that expressions (A3) and (A4) represent the same mutual inductance. For example, for the set of data: $l=1 \mathrm{~m}, d=3 \mathrm{~mm}, k$ $=0.2 \mathrm{~m}, x_{M}=2 \mathrm{~mm}, y_{M}=3 \mathrm{~mm}, z_{M}=0.3 \mathrm{~m}$, and $l_{M}=0.25 \mathrm{~m}$, the difference between (A3) and (A4) is only $0.0005 \%$. This proves without doubt that only the active filaments play a role and the rest of the MIDOT circuit is passive.

\section{Demonstration 2}

\section{a. A MIDOT probe provides very localized results}

To prove this it is necessary to calculate the mutual inductance between a MIDOT probe and a bifilar line of length 
equal to that of the active filaments and to compare this with the value when the bifilar line is much longer. The "local" mutual inductance is straightforwardly calculated from (A4) for $l=\mathrm{z}_{\mathrm{M}}$ and $k=0$. In most cases the differences can be neglected, e.g., for the set of data used above the difference between the real mutual inductance and the local mutual inductance is only $1.58 \%$. This extremely important result proves that only the part of the transmission line facing the MIDOT probe plays an important role in the measurement.

\section{Demonstration 3}

\section{a. A MIDOT probe does not require calibration}

As shown above, the MIDOT characteristics are $x_{M}, y_{M}$, $z_{M}$, and $l_{M}$. In the case of a strip-line, $x_{M}$ will take values that span the strip-line width $w$. As the line width is usually large, i.e., tens to hundreds of $\mathrm{mm}$, the precision of $x_{M}$ is not an issue. The same is valid for $z_{M}$ and $l_{M}$ : their values can be measured with accuracy better than $1 \%$. Since the MIDOT probe circuit can be easily positioned accurately in a plane parallel to the strip-line, the only issue remaining is the precision with which $y_{M}$ is known. For the set of data used above, a variation of $y_{M}$ by more than $300 \mu \mathrm{m}$ changes $M_{\text {MIDOT }}$ by less than $5 \%$. The required accuracy is certainly attainable in practice.

${ }^{1}$ R. S. Hawke, A. L. Brooks, C. M. Fowler, and D. R. Peterson, "Electromagnetic railgun launchers: Direct launch feasibility," AIAA J. 20(7), 978-985 (1982).

${ }^{2}$ A. Lefrançois, P.-Y. Chanal, G. Le Blanc, J. Petit, G. Avrillaud, and M. Delchambre, "High-velocity flyer-plate developments on two high-pulsed- power generators based on a strip-line design (GEPI and CEPAGE)," IEEE Trans. Plasma Sci. 39(1), 288-293 (2011).

${ }^{3}$ B. M. Novac, K. Omar, N. Graneau, I. R. Smith, and M. Sinclair, "Numerical modelling of a flyer plate electromagnetic accelerator," IEEE Trans. Plasma Sci. 40(10), 2300-2311 (2012).

${ }^{4}$ T. Ao, J. R. Asay, S. Chantrenne, M. R. Baer, and C. A. Hall, "A compact strip-line pulsed power generator for isentropic compression experiments," Rev. Sci. Instrum. 79(1), 013903 (2008).

${ }^{5}$ R. E. Reinovsky, W. L. Baker, Y. G. Chen, J. Holmes, and E. A. Lopez, "Shiva Star inductive compression system," in Proceedings of the 4th IEEE International Pulsed Power Conference (IEEE, 1983), pp. 197-201.

${ }^{6} \mathrm{H}$. Knoepfel, Pulsed High Magnetic Fields (North-Holland Publishing Company, London, 1970)

${ }^{7}$ O. Liebfried, M. Schneider, M. J. Loeffler, S. Balevičius, N. Žurauskienė, and V. Stankevič, "Measurement of the magnetic field distribution in railguns using CMR-B-scalar sensors," Acta Phys. Pol., A 115(6), 1125-1127 (2009).

${ }^{8}$ M. Schneider, O. Liebfried, V. Stankevič, S. Balevičius, and N. Žurauskiené, "Magnetic diffusion in railguns: Measurements using CMR-based sensors," IEEE Trans. Magn. 45(1), 430-435 (2009).

${ }^{9}$ Stanford Research Systems, DG535 Digital Delay Generator, http://www. thinksrs.com/products/DG535.htm.

${ }^{10}$ Pearson Electronics, Inc., Current Monitor Model 2877, http://www. pearsonelectronics.com/pdf/2877.pdf.

${ }^{11} \mathrm{PMK}$, Passive high voltage probe type PHV1000-RO, 4 kV, 400 MHz, 50 $\mathrm{M} \Omega, 7.5 \mathrm{pF}$, used with a BNC female adapter.

${ }^{12}$ B. M. Novac, M. Istenic, J. Luo, I. R. Smith, J. Brown, M. Hubbard, P. Appelgren, M. Elfsberg, T. Hurtig, C. Möller, A. Larsson, and S. E. Nyholm, "A 10-GW pulsed power supply for HPM sources," IEEE Trans. Plasma Sci. 34(5), 1814-1821 (2006).

${ }^{13}$ R. Bealing and P. G. Carpenter, "A compact 2MJ capacitor bank," AWRE Report No. 02/76, 1976.

${ }^{14} \mathrm{~F}$. W. Grover, Inductance Calculations: Working Formulas and Tables (Dover, New York, 1946).

${ }^{15}$ K. J. Binns, P. J. Lawrenson, and C. W. Trowbridge, The Analytical and Numerical Solution of Electric and Magnetic Fields (John Wiley \& Sons, New York, 1992). 